\title{
40 How obesity causes type 2 diabetes
}

Worldwide, the number of adults with type 2 diabetes has more than doubled since 1980 (Danaei et al. 2011). Why obesity is the main cause for this can be plausibly explained by knowledge of the underlying molecular mechanisms. For example, the tumor necrosis factor $\alpha$ produced in the excess fat deposits, the peptide hormone resistin as well as the fetuin-A given off by fatty livers create an insulin resistance in the muscle cells by blocking the insulin receptors on the cell surfaces. This insulin resistance, together with the associated reduced production of insulin by the $\beta$-cells in the pancreas that then usually takes place after a longer period, is what causes type 2 diabetes.
Type 2 diabetes is the pacing car for cardiovascular diseases and cancer.

Interleukin 6 , also produced by the adipose tissue in chronic inflammation and obesity, is a further risk factor because it stimulates glucose formation in the liver and thereby initiates additional insulin consumption. By contrast, adiponectin has previously been thought to be a protection factor, because higher levels of adiponectin are often found when any type 2 diabetes pathology is absent ( $\mathrm{Li}$ et al. 2009). This initially appears to make sense given that adiponectin stimulates the insulin-independent glucose uptake in the muscle cells and inhibits glucose release in the liver. In actuality, no causal connection exists here. Targeted rises in the adiponectin level do not lower the risk of developing type 2 diabetes (Yaghootkar et al. 2013).

As little as a 10-percent weight reduction markedly improves insulinsensitivity because the disruption in the normal manifestation of the insulin receptors on the muscle cells can be reversed quickly through persistent work by the muscle.

Fig. 40.1 Molecular mechanism of the development of type 2 diabetes. TNFa tumor necrosis factor a, IL-6 interleukin 6 